\title{
Roles of deformation and neutron excess on the giant monopole resonance in neutron-rich $\mathrm{Zr}$ isotopes
}

\author{
Kenichi Yoshida \\ RIKEN Nishina Center for Accelerator-Based Science, Wako, Saitama 351-0198, Japan
}

(Dated: October 29, 2018)

\begin{abstract}
We investigate the roles of deformation on the giant monopole resonance (GMR), particularly the mixing of the giant quadrupole resonance (GQR) and the effects of the neutron excess in the well-deformed nuclei around ${ }^{110} \mathrm{Zr}$ and in the drip-line nuclei around ${ }^{140} \mathrm{Zr}$ by means of the deformed quasiparticle-random-phase-approximation employing the Skyrme and the local pairing energy-density functionals. It is found that the isoscalar (IS) GMR has a two-peak structure, the lower peak of which is associated with the mixing between the ISGMR and the $K^{\pi}=0^{+}$component of the ISGQR. The transition strengths of the lower peak of the ISGMR grows as the neutron number increases. In the drip-line nuclei, the neutron excitation is dominant over the proton excitation. We find for an isovector (IV) excitation the GMR has a four-peak structure due to the mixing of the IS and IV modes as well as the mixing of the $K^{\pi}=0^{+}$component of the IVGQR. Besides the GMR, we find the threshold strength generated by neutrons only.
\end{abstract}

PACS numbers: 21.10.Re; 21.10.Pc; 21.60.Ev; 21.60.Jz; 24.30.Cz

\section{INTRODUCTION}

Nuclei far from the stability has been one of the major interests in nuclear structure physics because exploring the multipole responses in unstable nuclei can provide information on collective excitations in asymmetric nuclear systems. The surface structure of neutron-rich nuclei is quite different from that of stable nuclei, because of the presence of the loosely bound neutrons. Since collective excitations are sensitive to the surface structure, we can expect new kinds of exotic excitation modes to appear in neutron-rich nuclei.

An example of such an exotic excitation mode in unstable nuclei is the pygmy dipole resonance (PDR) appearing in the low energy region of the giant dipole resonance (GDR) [1]. The PDR consists of the isoscalar (IS) and isovector (IV) characters, and they are thought to be strongly mixed [2]. Strong mixing of the IS and IV character leads to a possible emergence of the neutronor proton-dominant giant resonance (GR) in unstable nuclei.

GRs are typical collective modes of excitation in nuclei. Effects of the nuclear deformation on the GRs has been investigated for long both experimentally and theoretically [3]. The deformation splitting of the GDR has been well established. This is due to the oscillation along the major- and minor-axis 4]. For the GRs with higher multipolarity, the deformation splitting is less pronounced [3]. Deformation effects on the GMR has been discussed soon after the GMR was established [5].

Collective excitations such as GRs and low-lying vibrational modes are described microscopically by the self-consistent Hartree-Fock (HF) plus the random-phase approximation (RPA). There have been many attempts to investigate the new kinds of collective excitations in unstable nuclei [1, 6, 7]. They are however largely restricted to spherical systems, and the collective excitation modes in deformed neutron-rich nuclei remain mostly un- explored.

Role of deformation on GRs has been studied by means of the deformed quasiparticle-RPA (QRPA) employing the Gogny interaction in the light mass region [8]. Using Skyrme functionals, GRs in heavy systems have been investigated by means of the RPA, where the separable approximation is employed for the residual interaction [9].

The ground-state properties, deformation and superfluidity, in neutron-rich Zr isotopes up to the drip line have been studied [10, 11], and it is predicted that ${ }^{112} \mathrm{Zr}$ has large deformation, and that the drip-line nuclei are also strongly deformed. In the present article, we investigate the GMR in neutron-rich medium-mass nuclei around ${ }^{110} \mathrm{Zr}$ and the drip-line nuclei around ${ }^{140} \mathrm{Zr}$ with paying attention to the deformation effects and the mixing of the IS and IV characters. To this end, we use the newly-developed parallelized HFB and deformed-QRPA calculation code [12].

The article is organized as follows: In Sec. III the deformed Skyrme-HFB + QRPA method is recapitulated. In Sec. [II] results of the numerical analysis of the giant resonances in deformed neutron-rich $\mathrm{Zr}$ isotopes are presented. Finally, summary is given in Sec. IV

\section{MODEL}

A detailed discussion of the deformed Skyrme-HFB + QRPA can be found in Refs. [13]. Therefore, we just briefly recall the outline of the calculation scheme.

In order to describe simultaneously the nuclear deformation and the pairing correlations including the unbound quasiparticle states, we solve the HFB equations [14, 15] in coordinate space using cylindrical coordinates $\boldsymbol{r}=(\rho, z, \phi)$. We assume axial and reflection symmetries. For the mean-field Hamiltonian $h$, we employ the $\mathrm{SkM}^{*}$ interaction [16]. The pairing field is treated by 
using the density-dependent contact interaction [17]

$$
v_{\text {pair }}\left(\boldsymbol{r}, \boldsymbol{r}^{\prime}\right)=\frac{1-P_{\sigma}}{2}\left[t_{0}^{\prime}+\frac{t_{3}^{\prime}}{6} \varrho_{0}^{\gamma}(\boldsymbol{r})\right] \delta\left(\boldsymbol{r}-\boldsymbol{r}^{\prime}\right)
$$

where $\varrho_{0}(\boldsymbol{r})$ denotes the isoscalar density of the ground state and $P_{\sigma}$ the spin exchange operator. Assuming timereversal symmetry and reflection symmetry with respect to the $x-y$ plane, we have to solve for positive $\Omega$ and positive $z$ only, $\Omega$ being the $z$-component of the angular momentum $j$. We use a lattice mesh size $\Delta \rho=\Delta z=0.6$ $\mathrm{fm}$ and a box boundary condition at $\rho_{\max }=14.7 \mathrm{fm}$, $z_{\max }=14.4 \mathrm{fm}$. The differential operators are represented by use of the 11-point formula of finite difference method. Since the parity and $\Omega$ are good quantum numbers in the present calculation scheme, we have only to diagonalize the HFB Hamiltonian for each $\Omega^{\pi}$ sector. The quasiparticle energy is cut off at $E_{\mathrm{qp}, \text { cut }}=60 \mathrm{MeV}$ and the quasiparticle states up to $\Omega^{\pi}=23 / 2^{ \pm}$are included.

The pairing strength parameter $t_{0}^{\prime}$ is determined so as to approximately reproduce the experimental pairing gap of ${ }^{120} \mathrm{Sn}\left(\Delta_{\exp }=1.245 \mathrm{MeV}\right)$. The strength $t_{0}^{\prime}=-240 \mathrm{MeV} \mathrm{\textrm {fm } ^ { 3 }}$ for the mixed-type interaction $\left(t_{3}^{\prime}=-18.75 t_{0}^{\prime}\right)$ [18] with $\gamma=1$ leads to the neutron pairing gap $\left\langle\Delta_{\nu}\right\rangle=1.20 \mathrm{MeV}$ in ${ }^{120} \mathrm{Sn}$.

Using the quasiparticle basis obtained as a selfconsistent solution of the HFB equations, we solve the QRPA equation in the matrix formulation [19]

$$
\sum_{\gamma \delta}\left(\begin{array}{cc}
A_{\alpha \beta \gamma \delta} & B_{\alpha \beta \gamma \delta} \\
-B_{\alpha \beta \gamma \delta} & -A_{\alpha \beta \gamma \delta}
\end{array}\right)\left(\begin{array}{c}
X_{\gamma \delta}^{i} \\
Y_{\gamma \delta}^{i}
\end{array}\right)=\hbar \omega_{i}\left(\begin{array}{c}
X_{\alpha \beta}^{i} \\
Y_{\alpha \beta}^{i}
\end{array}\right) .
$$

The residual interaction in the particle-hole (p-h) channel appearing in the QRPA matrices $A$ and $B$ is derived from the Skyrme density functional. We neglect the Coulomb interaction to reduce the computing time in the QRPA calculation. We expect that the two-body Coulomb interaction plays only a minor role 20, 21]. We drop the socalled " $J$ " term $C_{t}^{T}$ both in the HFB and QRPA calculations. The residual interaction in the particle-particle (p-p) channel is derived from the pairing functional constructed from the density-dependent contact interaction (11). Furthermore, we introduce a cut-off for the twoquasiparticle excitation energy as $60 \mathrm{MeV}$.

Since the full self-consistency between the static meanfield calculation and the dynamical calculation is broken by the above neglected term and the severe cut-off for the two-quasiparticle excitations, the spurious states may have finite excitation energies. In the present calculation, the spurious states appear at $0.58 \mathrm{MeV}$ and $0.53 \mathrm{MeV}$ for the $K^{\pi}=0^{+}$and $1^{+}$states, respectively in ${ }^{110} \mathrm{Zr}$. We assume the contamination of the spurious component in GRs to be small because the GRs are well apart from the spurious states.

In the present calculation, the number of twoquasiparticle excitations for the $K^{\pi}=0^{+}$excitation in ${ }^{110} \mathrm{Zr}\left({ }^{140} \mathrm{Zr}\right)$ is about 36000 (46 200). For the calculation of the matrix elements and diagonalization, it takes
TABLE I: Ground state properties of $100,108,110,112,136,138,140 \mathrm{Zr}$ obtained by the deformed HFB calculation with the $\mathrm{SkM}^{*}$ interaction and the mixed-type pairing interaction. Chemical potentials (in $\mathrm{MeV}$ ), deformation parameters, intrinsic quadrupole moments (in $\mathrm{fm}^{2}$ ), average pairing gaps (in $\mathrm{MeV}$ ), root-mean-square radii (in

\begin{tabular}{|c|c|c|c|c|c|c|c|}
\hline & ${ }^{100} \mathrm{Zr}$ & ${ }^{108} \mathrm{Zr}$ & ${ }^{110} \mathrm{Zr}$ & ${ }^{112} \mathrm{Zr}$ & ${ }^{136} \mathrm{Zr}$ & ${ }^{138} \mathrm{Zr}$ & ${ }^{140} \mathrm{Zr}$ \\
\hline$\lambda_{\nu}$ & -6.81 & -4.57 & -4.31 & -3.76 & -0.51 & -0.36 & -0.10 \\
\hline$\lambda_{\pi}$ & -10.6 & -13.6 & -14.4 & -15.0 & -22.3 & -22.6 & -22.8 \\
\hline$\beta_{2}^{\nu}$ & 0.38 & 0.37 & 0.37 & 0.39 & 0.28 & 0.29 & 0.31 \\
\hline$\beta_{2}^{\pi}$ & 0.41 & 0.42 & 0.42 & 0.43 & 0.32 & 0.33 & 0.36 \\
\hline$\langle Q\rangle_{\nu}$ & 602 & 791 & 753 & 849 & 947 & 1023 & 1135 \\
\hline$\langle Q\rangle_{\pi}$ & 403 & 429 & 432 & 456 & 355 & 374 & 409 \\
\hline$\langle\Delta\rangle_{\nu}$ & 0.00 & 0.61 & 0.55 & 0.00 & 0.70 & 0.64 & 0.53 \\
\hline$\langle\Delta\rangle_{\pi}$ & 0.32 & 0.00 & 0.00 & 0.00 & 0.36 & 0.36 & 0.36 \\
\hline$\sqrt{\left\langle r^{2}\right\rangle_{\nu}}$ & 4.60 & 4.77 & 4.82 & 4.88 & 5.29 & 5.34 & 5.39 \\
\hline$\sqrt{\left\langle r^{2}\right\rangle_{\pi}}$ & 4.43 & 4.51 & 4.53 & 4.56 & 4.69 & 4.72 & 4.75 \\
\hline
\end{tabular}
$\mathrm{fm}$ ) for neutrons and protons are listed.

about 346 (570) CPU hours and 184 (310) CPU hours, respectively.

\section{RESULTS AND DISCUSSION}

We summarize in Table \the ground state properties. The neutron-rich $\mathrm{Zr}$ isotopes under investigation are prolately deformed. This is consistent with the results calculated using the Skyrme SLy4 interaction [10], and we also find that ${ }^{112} \mathrm{Zr}$ has largest deformation. Both neutrons and protons are unpaired in this nucleus. This calculation indicates that ${ }^{140} \mathrm{Zr}$ is located very close to the drip line; the chemical potential is only $-0.1 \mathrm{MeV}$. The neutron skin well develops in this nucleus; the difference of the radii of neutrons and protons is $0.64 \mathrm{fm}$.

Figures 1 and 2 show the isoscalar (IS) and isovector (IV) monopole and quadrupole transition-strength distributions in ${ }^{90,100,108,110,112} \mathrm{Zr}$ and in ${ }^{136,138,140} \mathrm{Zr}$. Here we define these operators as

$$
\begin{aligned}
\hat{F}_{\lambda=0}^{\tau=0} & =\sum_{\tau_{z}=1,-1} \int d \boldsymbol{r} r^{2} \hat{\psi}_{\tau_{z}}^{\dagger}(\boldsymbol{r}) \hat{\psi}_{\tau_{z}}(\boldsymbol{r}), \\
\hat{F}_{\lambda=0}^{\tau=1} & =\sum_{\tau_{z}=1,-1} \int d \boldsymbol{r} \tau_{z} r^{2} \hat{\psi}_{\tau_{z}}^{\dagger}(\boldsymbol{r}) \hat{\psi}_{\tau_{z}}(\boldsymbol{r}), \\
\hat{F}_{\lambda=2, K}^{\tau=0} & =\sum_{\tau_{z}=1,-1} \int d \boldsymbol{r} r^{2} Y_{\lambda K}(\hat{r}) \hat{\psi}_{\tau_{z}}^{\dagger}(\boldsymbol{r}) \hat{\psi}_{\tau_{z}}(\boldsymbol{r}), \\
\hat{F}_{\lambda=2, K}^{\tau=1} & =\sum_{\tau_{z}=1,-1} \int d \boldsymbol{r} \tau_{z} r^{2} Y_{\lambda K}(\hat{r}) \hat{\psi}_{\tau_{z}}^{\dagger}(\boldsymbol{r}) \hat{\psi}_{\tau_{z}}(\boldsymbol{r}) .
\end{aligned}
$$

A nucleon creation operator $\hat{\psi}^{\dagger}(\boldsymbol{r})$ at the position $\boldsymbol{r}$ is written in terms of the quasiparticle wavefunctions. 


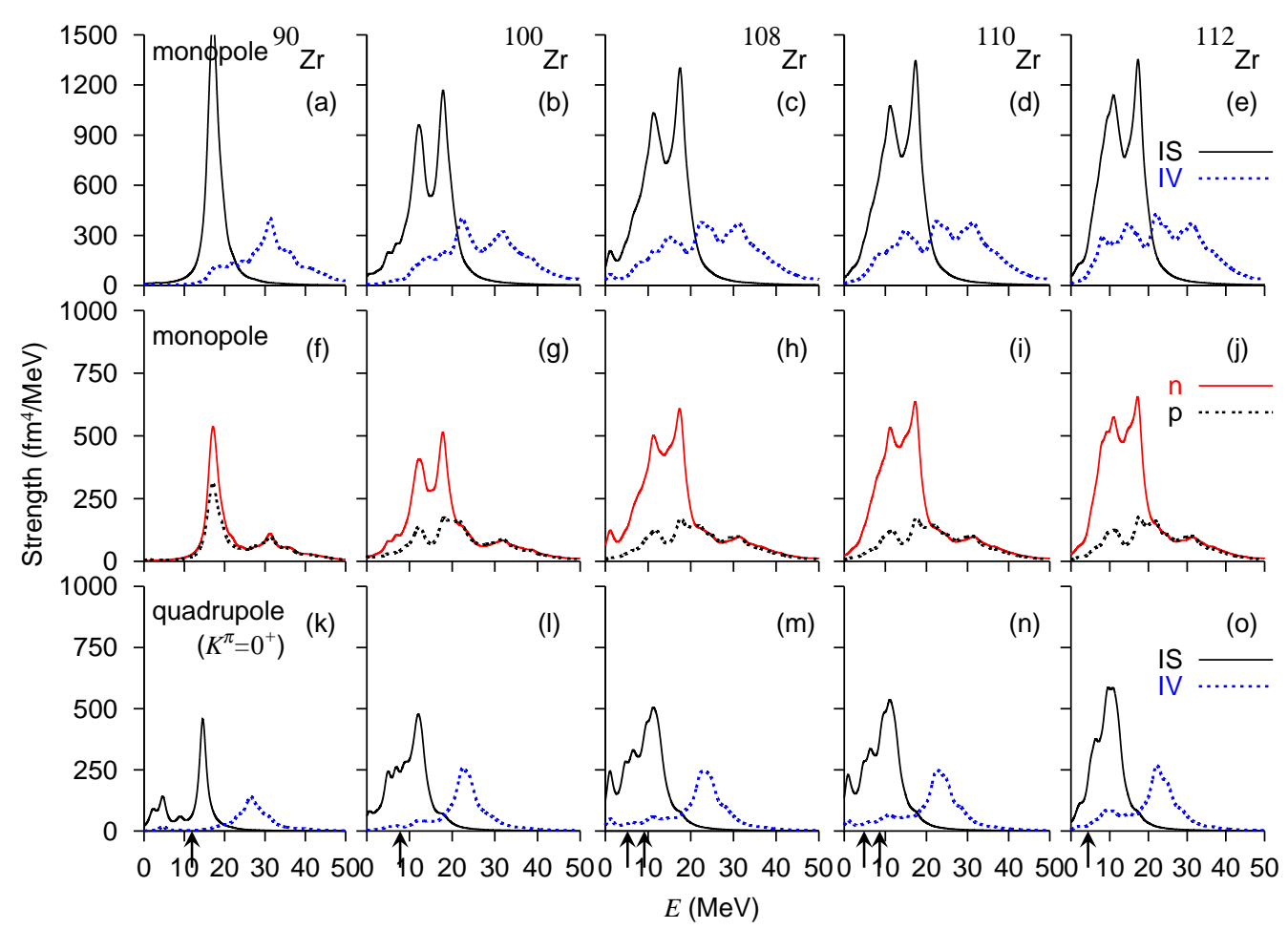

FIG. 1: (Color online) Isoscalar (IS) and isovector (IV) monopole and quadrupole transition strengths in ${ }^{90,100,108,110,112} \mathrm{Zr}$. The neutron and proton transition strengths are also shown for the monopole excitation. The arrows indicate the one-quasineutron continuum threshold $E_{\mathrm{th}, 1 n}=|\lambda|+\min E_{\alpha}$ and the two-quasineutron continuum threshold $E_{\mathrm{th}, 2 n}=2|\lambda|$. Shown is only the one-neutron continuum threshold for ${ }^{90,100,112} \mathrm{Zr}$, the normalfluid systems.

The strength function is calculated as

$$
S_{\lambda}^{\tau}(E)=\sum_{i} \sum_{K} \frac{\Gamma / 2}{\pi} \frac{\left|\left\langle i\left|\hat{F}_{\lambda K}^{\tau}\right| 0\right\rangle\right|^{2}}{\left(E-\hbar \omega_{i}\right)^{2}+\Gamma^{2} / 4}
$$

The smearing width $\Gamma$ is used to remove the spurious oscillation in the strength functions due to the box boundary condition, and it is set to $\Gamma=2 \mathrm{MeV}$ in the case of the box size of $15 \mathrm{fm}$ [22].

The ISGMR has a two-peak structure in the deformed nuclei. The higher-energy peak of the IS monopole excitation seen around $20 \mathrm{MeV}$ is identified as a primal ISGMR because the ISGMR in ${ }^{90} \mathrm{Zr}$ is located at around $18 \mathrm{MeV}$, where we have only one peak.

To understand the origin of the lower-energy peak around $10 \mathrm{MeV}$, we show in Figs. 1(k)-1(o) the $K^{\pi}=0^{+}$ component of the quadrupole transition strength. At the same energy region where the lower-energy peak of the ISGMR appears, we can see a peak structure. It is noted here that the neutron-rich Zr isotopes under investigation are prolately deformed, so the giant quadrupole resonance (GQR) splits into three resonances of the $K^{\pi}=0^{+}, 1^{+}$, and $2^{+}$components, and the resonance peak of the $K^{\pi}=0^{+}$component lowers in energy.

We are going to discuss in detail the deformation splitting of the ISGMR. Figure 3(a) shows the mean energy of the lower peak and the upper peak of the ISGMR.
The mean energy is evaluated as $m_{1} / m_{0}$, the ratio of the energy-weighted sum to the non energy-weighted sum. The energy interval is set to $5 \mathrm{MeV}<\hbar \omega_{i}<E_{c}$ and $E_{c}<\hbar \omega_{i}<25 \mathrm{MeV}$ for the lower and upper peaks. The value $E_{c}$ is set to $15 \mathrm{MeV}$ and $14 \mathrm{MeV}$ for ${ }^{100} \mathrm{Zr}$ and $108,110,112 \mathrm{Zr}$, respectively. We change $E_{c}$ associated with the mass number because the resonance energy decreases as the mass number increases. The values obtained in the scaling model 23] are also shown in Fig. 3.

The results of the present calculation are not very different from those obtained in the scaling model, with the exception that the scaling model overestimates the energy of the compressible mode [23]. As the system is deformed from ${ }^{90} \mathrm{Zr}$ to ${ }^{100} \mathrm{Zr}$, the excitation energy of the upper peak is upward-shifted due to the coupling with the $K^{\pi}=0^{+}$component of the ISGQR. Then, as the mass number increases from $A=100$ to 112 , the excitation energy is downward-shifted. The difference in the excitation energy of the upper peak in ${ }^{100} \mathrm{Zr}$ and in ${ }^{112} \mathrm{Zr}$ is $0.7 \mathrm{MeV}$. This energy difference is understood by the systematic trend of the excitation energy $E_{x} \propto A^{-1 / 3}$.

The lower peak of the ISGMR in deformed systems originates from the ISGQR in the spherical limit. The excitation energy of the lower peak in the scaling model agrees well with that obtained in the present calculation.

Figure 3(b) shows the fraction of the EWSR value for the lower and upper peaks of the ISGMR. The en- 


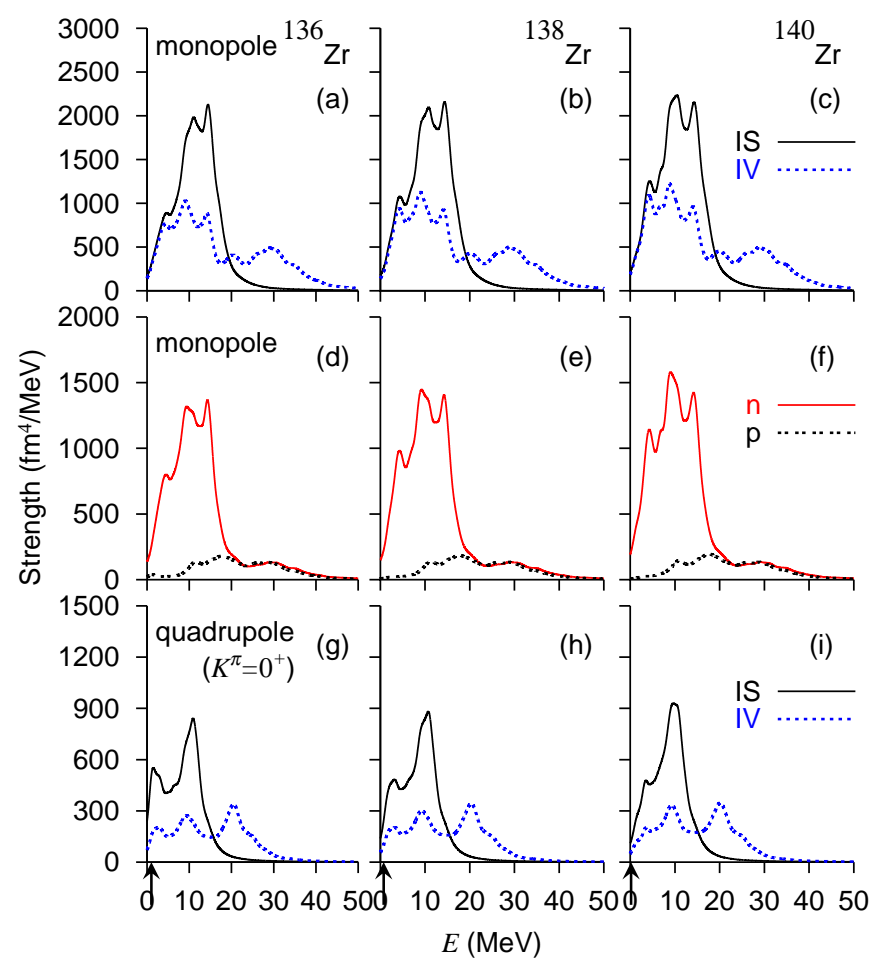

FIG. 2: (Color online) Same as Fig. 11 but in ${ }^{136,138,140} \mathrm{Zr}$.
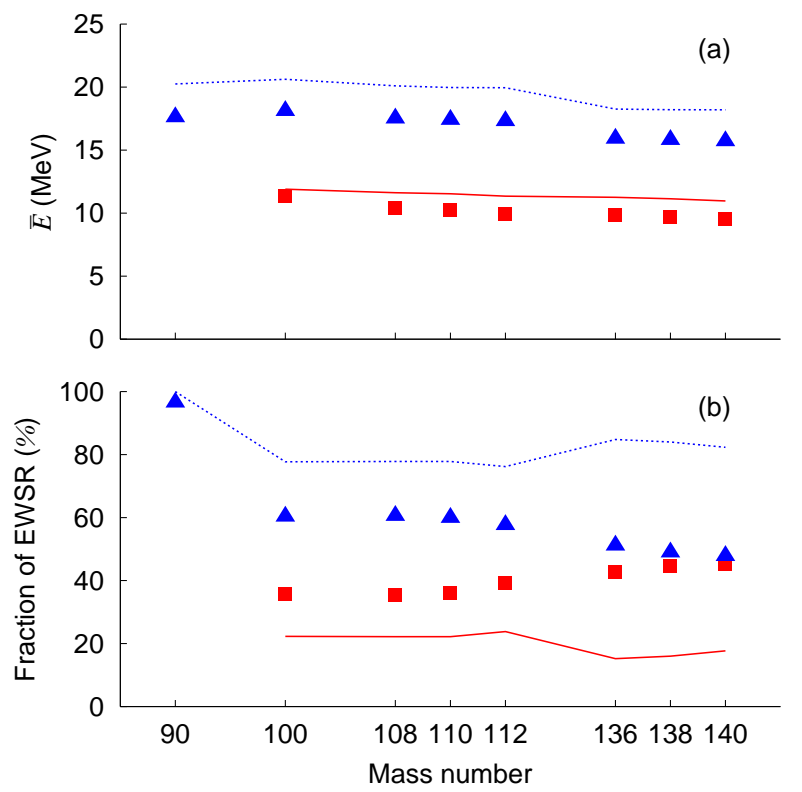

FIG. 3: (Color online) (a) Mean energy of the ISGMR in $\mathrm{Zr}$ isotopes. The energy of the upper and lower peaks are denoted by triangle and square, respectively. (b) Fraction of the EWSR value for the upper and lower peaks. The mean energy of the upper and lower peaks is calculated as $m_{1} / m_{0}$ for the energy interval of $5 \mathrm{MeV}<E<E_{c}$ and $E_{c}<E<25$ $\mathrm{MeV}$, and the fraction is calculated for each interval. The lines represent the results obtained in the scaling model [23].

TABLE II: Sum of the transition strengths of neutrons and protons $M_{\nu}^{2}, M_{\pi}^{2}$ (in $10^{3} \mathrm{fm}^{4}$ ) and $M_{\nu} / M_{\pi}$ divided by the neutron and proton numbers for the lower and upper peaks of the ISGMR.

\begin{tabular}{ccccccc}
\hline \hline \multicolumn{3}{c}{ Lower peak } & \multicolumn{3}{c}{ Upper peak } \\
& $M_{\nu}^{2}$ & $M_{\pi}^{2}$ & $\frac{M_{\nu} / M_{\pi}}{N / Z}$ & $M_{\nu}^{2}$ & $M_{\pi}^{2}$ & $\frac{M_{\nu} / M_{\pi}}{N / Z}$ \\
\cline { 6 - 8 } & & & & 2.62 & 1.70 & 0.99 \\
${ }^{90} \mathrm{Zr}$ & & & & & \\
${ }^{100} \mathrm{Zr}$ & 2.33 & 0.75 & 1.18 & 2.75 & 1.44 & 0.92 \\
${ }^{108} \mathrm{Zr}$ & 3.53 & 0.76 & 1.27 & 3.68 & 1.43 & 0.94 \\
${ }^{110} \mathrm{Zr}$ & 3.64 & 0.77 & 1.24 & 3.85 & 1.48 & 0.92 \\
${ }^{112} \mathrm{Zr}$ & 4.50 & 0.85 & 1.28 & 3.93 & 1.54 & 0.89 \\
${ }^{136} \mathrm{Zr}$ & 9.35 & 0.60 & 1.64 & 5.82 & 1.78 & 0.75 \\
${ }^{138} \mathrm{Zr}$ & 10.2 & 0.65 & 1.62 & 5.83 & 1.82 & 0.73 \\
${ }^{140} \mathrm{Zr}$ & 10.8 & 0.72 & 1.55 & 6.05 & 1.86 & 0.72 \\
\hline \hline
\end{tabular}

transition strengths in the low energy region, we show in the middle panels of Figs. 1 and 2 the neutron and proton monopole transition strengths. It is seen that the neutron excitation plays a dominant role in increasing the IS transition strengths in the energy region around $10 \mathrm{MeV}$.

Table II summarizes the contribution of the neutrons and protons to the lower and upper peaks of the ISGMR. We can see the effect of the neutron excess on the GMR

To investigate the origin of the enhancement of the 
quantitatively from this Table. Listed in this Table are the sum of the transition strengths of neutrons $M_{\nu}^{2}$ and protons $M_{\pi}^{2}$ in the energy intervals of $5 \mathrm{MeV}<\hbar \omega_{i}<E_{c}$ and $E_{c}<\hbar \omega_{i}<25 \mathrm{MeV}$. If the neutrons and protons coherently contribute to the excitation mode, the ratio of the matrix elements of neutrons to protons may approach the ratio of the neutron number to the proton number.

The ratio of the matrix elements divided by the ratio of neutron to proton numbers, $\left(M_{\nu} / M_{\pi}\right) /(N / Z)$, for the lower peak increases in the drip line nuclei. On the other hand, the quantity $\left(M_{\nu} / M_{\pi}\right) /(N / Z)$ for the upper peak decreases in the drip line nuclei. Thus, the neutron excitation concentrates particularly in the energy region of the lower peak of the ISGMR. Besides the GMR, we can see appearance of the threshold strength consisting of neutron excitations only in the drip-line nuclei. This fact is similar to the findings of Ref. 24] in ${ }^{60} \mathrm{Ca}$.

We are going to move on to the discussion on the properties of the IVGMR. In ${ }^{90} \mathrm{Zr}$, the mean energy of the IVGMR is $31.6 \mathrm{MeV}$. The hydrodynamical model taking a surface effect into account [25] predicts the excitation energy of the IVGMR to be

$$
E_{x} \simeq 88 A^{-1 / 6}\left(1+\frac{14}{3} A^{-1 / 3}\right)^{-1 / 2} \mathrm{MeV} .
$$

This gives the energy of the IVGMR in ${ }^{90} \mathrm{Zr}$ to be about $29 \mathrm{MeV}$. This is consistent with the microscopically calculated energy.

The IVGMR in deformed neutron-rich nuclei has interesting features, where a four-peak structure appears as shown in Fig. 11. The four-peak structure is clearly seen especially in ${ }^{112} \mathrm{Zr}$.

The highest peak above $30 \mathrm{MeV}$ corresponds to a primal IVGMR by comparing the peak energy with that in ${ }^{90} \mathrm{Zr}$. We have a peak structure at $22-23 \mathrm{MeV}$ where the IVGQR is seen. Thus the second highest peak emerges associated with the coupling to the $K^{\pi}=0^{+}$component of the IVGQR.

Besides these two peaks, we can see two more peaks below $20 \mathrm{MeV}$. We can consider that they are brought to the energy region of the ISGMR due to the neutron excess. One is associated with the coupling to the ISGMR, and the other is due to the coupling to the $K^{\pi}=0^{+}$ component of the ISGQR.

In the drip-line nuclei, the transition strengths concentrate in the ISGMR region. We can still see a four-peak structure in ${ }^{136,138,140} \mathrm{Zr}$. The IV transition strengths become enhanced in the energy region where the neutron transition strengths increase. Therefore, we can see the threshold strength in the IV monopole strength distribution as well.

\section{SUMMARY}

We have investigated the deformation effects on the GMR by employing the newly developed parallelized QRPA calculation scheme. Associated with the deformation, the excitation modes with the angular momenta $l=0$ and $l=2$ can mix in the $K^{\pi}=0^{+}$channel, and accordingly we have obtained a two-peak structure of the ISGMR. The lower peak of the ISGMR is due to the coupling with the ISGQR. The deformation splitting is seen in nuclei from near-stability line to drip line.

The deformation splitting obtained in the present calculation is in fairly good agreement with the result of the scaling model, while the distribution of the strengths is quite different when the drip line is approached. The IS monopole transition strengths are enhanced in the energy region of the lower peak of the ISGMR. This is due to the concentration of the neutron transition strengths in the lower energy region.

We have found a complicated peak structure for the IVGMR in deformed neutron-rich nuclei, where a fourpeak structure appears. The highest peak corresponds to a primal IVGMR, and the second is associated with the coupling to the $K^{\pi}=0^{+}$component of the IVGQR, and the two more peaks are found in the ISGMR region associated with the neutron excess.

Besides the GMRs, the threshold strength is found in the drip-line nuclei. The strength consists of neutron strengths only. Since the low-lying $K^{\pi}=0^{+}$mode is quite sensitive to the shell structure and surface structure [26], we are going to investigate the microscopic structure of the low-lying $K^{\pi}=0^{+}$states with special attention to the coupling to the continuum states and the pairing correlations.

\section{Acknowledgments}

The author thanks K. Matsuyanagi for stimulating discussions and useful comments. He is supported by the Special Postdoctoral Researcher Program of RIKEN. The numerical calculations were performed on RIKEN Integrated Cluster of Clusters (RICC) and on the NEC SX-8 supercomputers at the Yukawa Institute for Theoretical Physics, Kyoto University and at the Research Center for Nuclear Physics, Osaka University.
[1] N. Paar, D. Vretenar, E. Khan and G. Colò, Rep. Prog. Phys. 70, 691 (2007).

[2] K. Yoshida, Phys. Rev. C 80, 044324 (2009).
[3] M. N. Harakeh and A. van der Wounde, Giant Resonances: Fundamental High-Energy Modes of Nuclear Excitation (Oxford, 2001). 
[4] A. Bohr and B. R. Motteleson, Nuclear Structure, vol. II (Benjamin, 1975; World Scientific, 1998).

[5] U. Garg et al., Phys. Rev. Lett. 45, 1670 (1980).

[6] M. Bender, P-H. Heenen, P-G. Reinhard, Rev. Mod. Phys. 75 (2003) 121.

[7] N. Paar, J. Phys. G: Nucl. Part. Phys. 37, 064014 (2010).

[8] S. Péru and H. Goutte, Phys. Rev. C 77, 044313 (2008).

[9] V. O. Nesterenko, W. Kleinig, J. Kvasil, P. Vasely, P. G. Reinhard, and D. S. Dolci, Phys. Rev. C 74, 064306 (2006).

[10] A. Blazkiewicz, V. E. Oberacker, and A. S. Umar, and M. Stoitsov, Phys. Rev. C 71, 054321 (2005).

[11] M. Stoitsov, N. Michel, and K. Matsuyanagi, Phys. Rev. C 77, 054301 (2008).

[12] K. Yoshida and T. Nakatsukasa, in preparation.

[13] K. Yoshida and N. V. Giai, Phys. Rev. C 78, 064316 (2008).

[14] A. Bulgac, Preprint No. FT-194-1980, Institute of Atomic Physics, Bucharest, 1980. arXiv:nucl-th/9907088

[15] J. Dobaczewski, H. Flocard and J. Treiner, Nucl. Phys. A422, 103 (1984).

[16] J. Bartel, P. Quentin, M. Brack, C. Guet, and H.-
B. Håkansson, Nucl. Phys. A386, 79 (1982).

[17] R. R. Chasman, Phys. Rev. C 14, 1935 (1976).

[18] K. Bennaceur and J. Dobaczewski, Comput. Phys. Commun. 168, 96 (2005).

[19] D. J. Rowe, Nuclear Collective Motion, (Methuen and Co. Ltd., 1970).

[20] J. Terasaki, J. Engel, M. Bender, J. Dobaczewski, W. Nazarewicz, and M. Stoitsov, Phys. Rev. C 71, 034310 (2005).

[21] S. Ebata, T. Nakatsukasa, T. Inakura, K. Yoshida, Y. Hashimoto, and K. Yabana, arXiv:1007.0785.

[22] T. Inakura, T. Nakatsukasa, and K. Yabana, Phys. Rev. C 80, 044301 (2009).

[23] S. Nishizaki and K. Andō, Prog. Theor. Phys. 73, 889 (1985).

[24] I. Hamamoto, H. Sagawa, and X. Z. Zhang, Phys, Rev. C 56, 3121 (1997).

[25] J. D. Bowman, E. Lipparini and S. Stringari, Phys. Lett. B197, 497 (1987).

[26] K. Yoshida and M. Yamagami, Phys. Rev. C 77, 044312 (2008). 